\title{
Cervical bone pain revealing a bone metastatic colon cancer: A case report
}

\author{
YAQOUB ALSHATTI ${ }^{1}$, SHUAIB ALQALLAF ${ }^{1}$, WASSIM ALLAHAM $^{2}$, SANDRA GRANIER $^{1}$, \\ PASCAL HAMMEL ${ }^{1}$, STEPHANE CULINE ${ }^{1,3}$ and MICHELE LAMURAGLIA ${ }^{1,4}$
}

${ }^{1}$ Oncology and ${ }^{2}$ Radiology Units, Assistance Publique-Hôpitaux de Paris, Beaujon Hospital, Clichy 92100; ${ }^{3}$ Oncology Unit, Assistance Publique-Hôpitaux de Paris, Saint Louis Hospital, Paris 75010; ${ }^{4}$ Laboratoire d' Imagerie Biomédicale (LIB), Sorbonne University, Centre National de la Recherche Scientifique, Institut National de la Santé et de la Recherche Médicale, Assistance Publique-Hôpitaux de Paris, Beaujon Hospital, Paris 75006, France

Received February 12, 2019; Accepted April 30, 2020

\section{DOI:10.3892/mco.2020.2072}

\begin{abstract}
Colorectal cancer (CRC) is one of the most common malignancies worldwide, with common sites of metastases including abdominal lymph nodes, the liver and lungs. Bone metastases are known to be relatively rare sites of metastasis. The present study reported a patient with advanced colorectal cancer and metastatic colorectal cancer (mCRC) with atypical metastases presentation. Bone mCRCs were not frequent and presented a poor prognosis in the present case. It was concluded that further studies are required to clarify the pathogenesis of bone metastases, to improve the management and treatment of patients.
\end{abstract}

\section{Introduction}

One of the most dreadful cancers globally is the colorectal cancer (CRC), it is rated at the third position among the prevalent causes of death in both men and women in the world. The common sites of metastases are abdominal lymph nodes (48.3\%), liver (57.6\%), and lungs (37.6\%) (1). Nonetheless, bone metastases are also diagnosed in about $3.7-11 \%$ in metastatic CRC (mCRC) patients (2) and 8.6-27\% are describe in autopsies series cases (3). We report a case of patient with mCRC with unhabitual bone metastases presentation.

\section{Case report}

A 56 year-old woman with a 6 month history of persistent and worsening neck pains on the right side without triggering factor, associated with paresthesia and distal right upper limb weakness. It was also noted that she had been

Correspondence to: Dr Michele Lamuraglia, Oncology Unit, Assistance Publique-Hôpitaux de Paris, Beaujon Hospital, 100 Bd Générale Leclaire, Clichy 92100, France

E-mail: michele.lamuraglia@aphp.fr

Key words: colorectal cancer, adenocarcinoma, bone metastasis smoking for 40 years. An examination was performed, which identified distal right upper limb deficit estimated at 4/5 on her interosseous muscles of the right hand. Additionally, a computerized tomography (CT) of the whole body showed revealed a multiple osteolytic bone lesions of the cranial vault and the axial and peripheral skeleton, a multi-nodular liver and both adrenal glands. Furthermore, a mass of the sigmoid colon was recognized, which was perceived to be the main tumor (Fig. 1). The subsequent testing of the blood exhibited high carcioembryonic agent (CEA) levels at 4,441 $\mu \mathrm{g} / 1$ as well as CA 19.9 serum levels of $85 \mathrm{kU} / 1$. Afterwards, a colonoscopy identified a stenosis, and an ulcerative circumferential abrasion of $15 \mathrm{~cm}$ after the anal margin. The histological analyses of the biopsies demonstrated that the colic adenocarcinoma was poorly differentiated (Ki-67:98\%, CK7 and CK20 negative; CDX2 affirmative) with NRAS, KRAS, BRAF and PIK3CA wild-type. Additionally, biopsies of the bone and the liver were conducted to confirm the analysis of the colonic metastasis.

The patient received only one cycle of the 5-FU, leucovorin in addition to oxaliplatin (FOLFOX) regime. This was followed by rapid deterioration of her clinical condition; she later succumbed to the disease 4 weeks after the diagnoses due to disease progression.

\section{Discussion}

A number of previous studies identified that bone metastatic colorectal cancer (mCRC) was infrequent $(1,2)$. The analysis in the United States National Cancer Registry between 2008 and 2013 reported advanced occurrence of the bone metastases CRC at an earlier age and in male patients. In addition, it was identified that bone metastases CRC was associated with liver metastasis and poor survival globally $(4,5)$. That given level of susceptibility may be due to the patterns of the blood movement commencing at the colorectal region proceeding to the portal system, as well as the molecular signaling leading to biological interactions between the body organs (6).

It is noteworthy that bone metastasis of CRC is a critical condition that affects the overall condition of the patient. Bone metastasis of CRC will advance during the period of diagnosis. Changes in the bowel habit are a common sign of the disease 

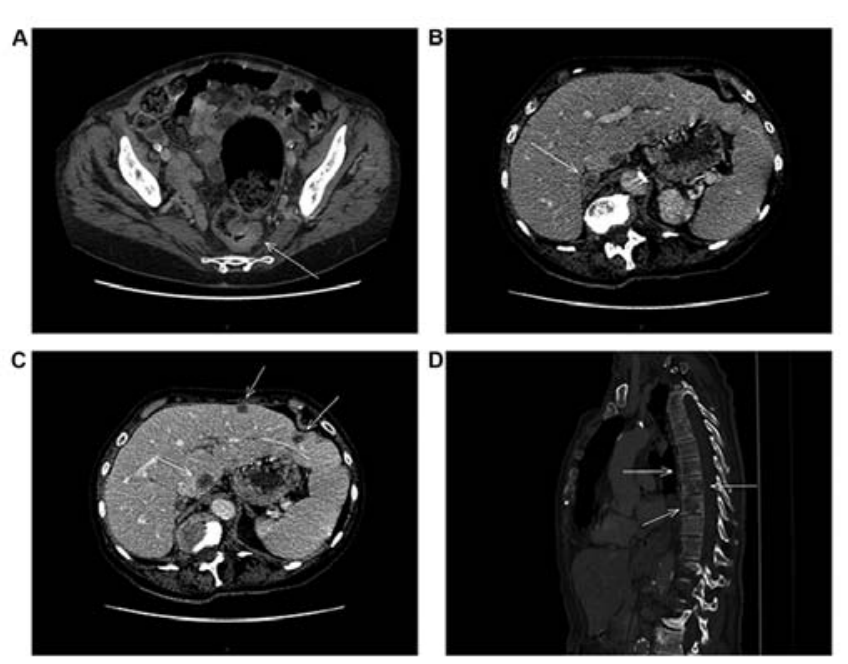

Figure 1. A total body diagnostic CT scan revealed metastasis. (A) Primary lesion of the sigmoid colon. (B) Right adrenal gland nodule. (C) Multi-nodular liver. (D) Multiple osteolytic lesions.

that may be associated with illness as the patient may experience poor nutrition. Katagiri et al (7) performed examinations of skeletal metastases of unidentified origin, and identified that primitive CRC (2/134 patients) was uncommon and was correlated with worse universal survival. Hanamura et al (8) described CRC as having a higher tendency of metastasis in the lungs, the liver and the lymph nodes at the abdominal regions, and rarely occurring in the bone marrow. There was a previous case report describing a 60 year-old man who had sigmoid CRC with bone marrow and lymph node metastasis, with a similar deterioration in health to the present patient. Table I presents 7 manuscripts that utilized chemotherapy with different overall survival (OS).

The exact chemotherapeutic treatments to counter metastases of the bone marrow have not yet been developed and there have been reports of poor prognosis among the majority of the patients with CRC. Methodological chemotherapy against metastasis $\mathrm{CRC}$ with bone marrow metastases has also proved ineffective (8).

However, the benefice to start the denosumab and bisphosphonates was not evident in our case, because of the rapid evolution of disease. Moreover, up to date, the benefit and effectiveness of using denosumab with bisphosphonates in cancer-related bone injuries has been demonstrated in literature. The metastasis of the bone in CRC is rare; however, has a poor prognosis and worsens the quality of life of the patients.

The molecular chromosomal variations can be more precise markers than clinicopathological characteristics to assess the prognosis of a patient with early and intermediate stage CRC. In the examination of 300 patients with stages I-III CRC, for instance, PIK3CA transformations predicted a poorer prognosis. However, this was only in the KRAS wild-type patients with CRC. Furthermore, PIK3CA transformation was correspondingly recognized as an autonomous biomarker for local recurrence amid stages I-III CRC; however, there was no convincing analytical significance for the KRAS transformations for early and intermediate stages of the CRC. Although a previous study demonstrated that NRAS transformations could predict a

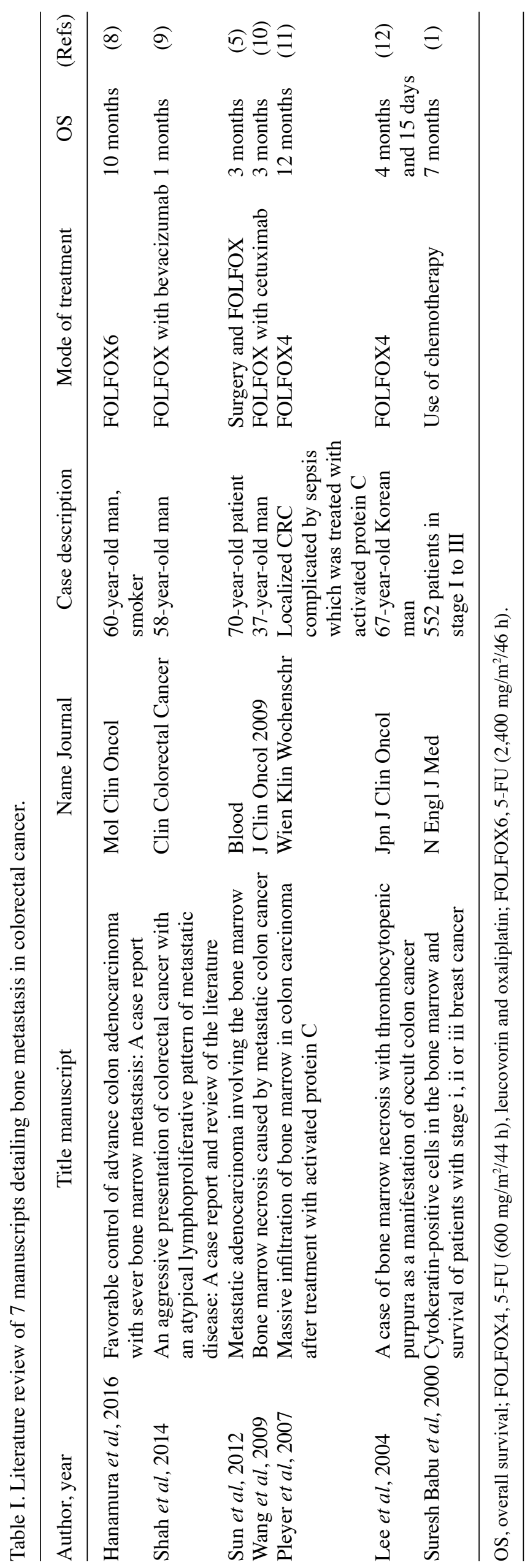


poor prognosis for patients with CRC with metastases (5), regardless of the discrepancies in the previous studies, it was suggested that the biological markers may predict the prognosis of a patient with $\mathrm{CRC}$ at different stages and may aid the individualized selection of adjuvant treatment for a patient after operation.

Sun et al (5) identified that in CRC, $>20$ genes and two RNAs have different expression patterns at the metastasis stage. In that previous study, the Gene Ontology pathway analysis demonstrated that microRNA-429 was involved in CRC. In addition to treatment decisions, future management should also be considered. Future studies should aim to perform an in-depth examination of the molecular structures coupled with genetic studies, to provide clarification of the pathogenesis of bone metastases. Furthermore, NRAS proteins, as well as the KRAS proteins, have been found to be homologous enzymes of the RAS protein group; the genetic assessments have elucidated variances. Additionally, the NRAS and the KRAS transformations have been found to have mutually exclusive mechanisms to prompt carcinogenesis; mutant KRAS prompts too much propagation and diversification of the human colonic epithelial cell streaks, whereas, NRAS mutants suppress apoptosis.

In conclusion, CRC is one of the most prevalent malignancies, causing a large number of deaths worldwide. The spread of the life-threatening neoplasms into the bone is not due to accidental progression; however, it involves a number of molecular interactions coordinated through composite exchanges between the neoplastic cells and their surroundings. Further molecular and genetic studies are needed to clarify the pathogenesis of bone metastases, which will be beneficial in guiding future management and treatment decisions.

\section{Acknowledgements}

Not applicable.

\section{Funding}

No funding was received.

\section{Availability of data and materials}

The datasets used and/or analyzed during the current study are available from the corresponding author on reasonable request.

\section{Authors' contributions}

YA and SA collected imaging data, collected measurements, analyzed the data and wrote the manuscript. WA performed imaging analyses. ML designed the current study and wrote the manuscript. SG, PH and SC collected the literature. The final version of the manuscript has been read and approved by all authors.

\section{Ethics approval and consent to participate}

The study protocol was approved by the local Ethics Committee and the Institutional Review Board of Assistance Publique-Hôpitaux de Paris, Beaujon Hospital.

\section{Patient consent for publication}

The patient consented to the publication of data and associated images.

\section{Competing interests}

The authors declare that they have no competing interests.

\section{References}

1. Suresh Babu MC, Garg S, Lakshmaiah KC, Babu KG, Kumar RV, Loknatha D, Abraham LJ, Rajeev LK, Lokesh KN, Rudresha AH and Rao SA: Colorectal cancer presenting as bone metastasis. J Cancer Res Ther 13: 80-83, 2017.

2. Kanthan R, Loewy J and Kanthan SC: Skeletal metastases in colorectal carcinomas: A saskatchewan profile. Dis Colon Rectum 42: 1592-1597, 1999.

3. Katoh M, Unakami M, Hara M and Fukuchi S: Bone metastasis from colorectal cancer in autopsy cases. J Gastroenterol 30: 615-618, 1995.

4. Gelson WT, Rimmer MJ, Landells W and Douds AC: Sacral metastasis as a presentation of colonic adenocarcinoma. J R Soc Med 100: 191-192, 2007.

5. Sun Y, Shen S, Tang H, Xiang J, Peng Y, Tang A, Li N, Zhou W, Wang Z, Zhang D, et al: MiR-429 identified by dynamic transcriptome analysis is a new candidate biomarker for colorectal cancer prognosis. OMICS 18: 54-64, 2014.

6. Schlüter K, Gassmann P, Enns A, Korb T, Hemping-Bovenkerk A, Hölzen J and Haier J: Organ-Specific metastatic tumor cell adhesion and extravasation of colon carcinoma cells with different metastatic potential. Am J Pathol 169: 1064-1073, 169.

7. Katagiri H, Takahashi M, Inagaki J, Sugiura H, Ito $\mathrm{S}$ and Iwata H: Determining the site of the primary cancer in patients with skeletal metastasis of unknown origin: A retrospective study. Cancer 86: 533-537, 1999.

8. Hanamura F, Shibata Y, Shirakawa T, Kuwayama M, Oda H, Ariyama H, Taguchi K, Esaki T and Baba E: Favorable control of advanced colon adenocarcinoma with severe bone marrow metastasis: A case report. Mol Clin Oncol 5: 579-582, 2016.

9. Shah SM, Rosenthal MH, Griffin GK, Jacobsen ED and McCleary NJ: Aggressive presentation of colorectal cancer with an atypical lymphoproliferative pattern of metastatic disease: A case report and review of the literature. Clin Colorectal Cancer 13: 5-11, 2014.

10. Wang YC, Chang PY and Yao NS: Bon arrow necrosis caused by metastatic colon cancer. J Clin Oncol 10: e48, 2009.

11. Pleyer L, Went P, Russ G, Prinz E, Faber V, Röwert HJ, Karlbauer R and Greil R: Massive infiltration of bone marrow in colon carcinoma after treatment with activated protein $\mathrm{C}$. Wien Klin Wochenschr 119: 254-258, 2007.

12. Lee JL, Lee JH, Kim MK, Cho HS, Bae YK, Cho KH, Bae SH, Ryoo HM, Lee KH and Hyun MS: A case of bone marrow necrosis with thrombotic thrombocytopenic purpura as a manifestation of occult colon cancer. Jpn J Clin Oncol 34: 476-480, 2004. 